\title{
Power and Bandwidth Minimization for Demand-Aware GEO Satellite Systems
}

\author{
Tedros Salih Abdu, Steven Kisseleff, Eva Lagunas and Symeon Chatzinotas \\ Interdisciplinary Centre for Security, Reliability and Trust (SnT), \\ University of Luxembourg, Luxembourg \\ Email: \{tedros-salih.abdu, steven.kisseleff, eva.lagunas, symeon.chatzinotas\}@uni.lu
}

\begin{abstract}
Smart radio resource allocation combined with the recent advances of digital payloads will allow to control the transmit power and bandwidth of the satellites depending on the demand and the channel conditions of users. The system flexibility is important not only to handle divergent demand requirements but also to efficiently utilize the limited and expensive satellite resources. In this paper, we propose a demand-aware smart radio resource allocation technique, where the transmit power and the bandwidth of the GEO satellite are minimized while satisfying the user demand. The formulated optimization problem is non-convex mixed-integer nonlinear program which is difficult to solve. Hence, we apply a quadratic transform to solve the problem iteratively. The numerical results showed that the proposed scheme outperforms the benchmark schemes in terms of bandwidth utilization while accurately providing capacity-ondemand.
\end{abstract}

Index Terms-GEO satellite, Smart radio resource allocation, Demand satisfaction, Bandwidth utilization, Quadratic transform

\section{INTRODUCTION}

The future satellites are expected to provide a high throughput to serve the increasing connectivity and traffic demands. Furthermore, the deployment of advanced digital payloads on these satellites will improve the control and the flexibility of resource allocation [1]. SES-17 [2] and EUTELSAT QUANTUM [3] are typical examples of such payloads. To fully utilize the scarce and expensive satellite resources as well as to support throughput-demanding services, smart radio resource allocation on the digital payloads is essential [4]. For this, a thorough research has been conducted to adaptively control the power and/or the bandwidth of the system with the aim of demand satisfaction. The resource allocation techniques developed so far can be divided into three categories:

1) Power Allocation: The system power is optimized based on the beams' requested demand while considering a uniform bandwidth allocation. In [5]-[7], a power allocation was studied to minimize the difference between the offered capacity and the requested demand. However, the bandwidth allocation is considered to be fixed where a conventional frequency reuse pattern is applied at the cost of spectrum utilization. In [8], a power optimization

This work has been supported by the Luxembourg National Research Fund (FNR) under the project FlexSAT (C19/IS/13696663) and the AFR Grant INSAT - "Power and Bandwidth Allocation for INterference-Limited SATellite Communication Systems". technique based on channel condition is considered, while in [9], power allocation with imperfect channel state information (CSI) is proposed. A distinct drawback of these works is that inter-beam interference has been neglected in these works. On the other hand, power allocation with the full frequency reuse scheme is considered in [10].

2) Bandwidth allocation: In this case, the system bandwidth is optimized assuming a fixed power to satisfy the requested demand. In this context, bandwidth assignment with the assumption of time sharing for Gaussian broadcast channels has been addressed in [11], [12]. In these works, the interference among the beams has not been considered again.

3) Joint power and bandwidth allocation: Both power and bandwidth are optimized to satisfy the requested demand. This joint allocation has been introduced in [13], [14], where orthogonal carriers are assigned within a cluster of beams. Moreover, a binary power allocation is considered for each carrier. Similarly, to minimize the unmet system capacity, an evolutionary algorithms have been proposed in [15], [16]. Furthermore, in [17], a joint carrier and power assignment is considered for demand matching. However, the optimization solution is infeasible when the system offered capacity is below the required demand. Hence, lower demand satisfaction is occurred.

In this paper, we consider power and bandwidth minimization for demand-aware GEO Satellite Systems. Hence, carrier and power are allocated wherever demand is needed. Furthermore, we consider an interference-limited GEO satellite system with a large number of beams sharing the spectrum resources. The formulated optimization problem is non-convex mixed-integer nonlinear program which is difficult to solve. Therefore, we apply a quadratic transform to solve the problem iteratively. The extensive numerical results show that the demand matching error and bandwidth utilization of the proposed strategy is substantially lower than that obtained with the benchmark schemes.

The rest of the paper is organized as follows. Section II describes the system model. The problem formulation and the proposed solution are provided in Section III and Section IV, respectively. The numerical results are presented in Section V and Section VI concludes the paper. 


\section{System Model}

Consider a broadband geostationary (GEO) satellite system with $N$ beams. The total bandwidth $B_{t o t}$ is subdivided into $K$ subcarrier bandwidth of $B_{s c}$ i.e. $B_{s c}=B_{t o t} / K$. Furthermore, we define a carrier allocation vector for carrier $k$ as $\mathbf{x}_{k}=\left[x_{k}[1], x_{k}[2], \ldots, x_{k}[N]\right]^{T}$, where $x_{k}[i]$ is binary-valued variable and $x_{k}[i]=1$ means that the carrier $k$ is allocated to the $i$ th beam. Similarly, we define the power allocation vector for carrier $k$ as $\mathbf{p}_{k}=\left[p_{k}[1], p_{k}[2], \ldots, p_{k}[N]\right]^{T}$, where $p_{k}[i]$ is the power required by the $i$ th beam to transmit in the $k$ th carrier. Assuming single user per beam, the signal-tointerference-plus-noise ratio (SINR) of the $i$ th beam over $k$ th carrier is given by

$$
\gamma_{i, k}\left(\mathbf{p}_{k}, \mathbf{x}_{k}\right)=\frac{g_{i, k}[i] p_{k}[i] x_{k}[i]}{\sum_{j=1, j \neq i}^{N} g_{i, k}[j] p_{k}[j] x_{k}[j]+\sigma^{2}},
$$

where $g_{i, k}[i]$ the channel power gain from the $i$ th beam over the $k$ th carrier to the $i$ th user and $\sigma^{2}$ is the noise power. Hence, the offered capacity at beam $i$ including all the possible available carriers is

$$
C_{i}=\sum_{k=1}^{K} B_{s c} \log _{2}\left(1+\gamma_{i, k}\left(\mathbf{p}_{k}, \mathbf{x}_{k}\right)\right)
$$

Finally, given the $i$ th beam demand $D_{i}$, the normalized unmet system capacity is given by

$$
C_{\text {unmet }}=\sum_{i=1}^{N} \max \left(1-C_{i} / D_{i}, 0\right) \text {. }
$$

\section{PROBLEM Formulation}

In this section, we formulate the resource optimization problem to minimize the power and the bandwidth of the system while closely matching per user demand. In this context, we select the unmet system capacity, the total transmit power, and the total number of utilized carriers as multi-objective functions for our problem formulation. Furthermore, in order to account for the practical modulation and coding schemes employed in traditional satellite communication systems, we impose a minimum SINR constraint for all beams. In addition, we introduce a binary variable $y_{k}$ to indicate whether $k$ th carrier is active, i.e. assigned by any user $\left(y_{k}=1\right)$ or not $\left(y_{k}=0\right)$. The optimization problem is given by

$\underset{\mathbf{y}_{k}, \widetilde{\mathbf{p}}_{k}, \forall_{k}}{\operatorname{minimize}} \sum_{i=1}^{N} \max \left(1-C_{i} / D_{i}, 0\right)+\sum_{k=1}^{K} y_{k}+\sum_{i=1}^{N} \sum_{k=1}^{K} \widetilde{p}_{k}[i]$ s.t.

$$
\begin{aligned}
& \text { T1 : } \gamma_{i, k}\left(\mathbf{x}_{k}, \widetilde{\mathbf{p}}_{k}\right) \geq x_{k}[i] \gamma_{\text {min }}, \forall_{i}, \forall_{k}, \\
& T 2: \sum_{i=1}^{N} \sum_{k=1}^{K} \widetilde{p}_{k}[i] \leq 1, \\
& T 3: \sum_{k=1}^{K} \widetilde{p}_{k}[i] P_{\text {total }} \leq P_{\text {max }}, \forall_{i},
\end{aligned}
$$

$$
\begin{aligned}
& T 4: y_{k} \in\{0,1\}, \forall_{k}, \\
& T 5: x_{k}[i] \in\{0,1\}, \forall_{i}, \forall_{k}, \\
& T 6: x_{k}[i] \leq y_{k}, \forall_{i}, \forall_{k}, \\
& T 7: P_{\text {total }} \widetilde{p}_{k}[i] \leq x_{k}[i] P_{\text {max }}, \forall_{i}, \forall_{k}, \\
& T 8: \widetilde{p}_{k}[i] \geq 0, \forall_{i}, \forall_{k},
\end{aligned}
$$

where $\sum_{k=1}^{K} y_{k}$ is the carrier utilization function and $\sum_{i=1}^{N} \sum_{k=1}^{K} \widetilde{p}_{k}[i]$ is the normalized power allocation function with $\widetilde{p}_{k}[i]=\frac{p_{k}[i]}{P_{\text {total }}}$. Note that we normalize the multi-objective function to avoid the mismatch unit and imbalance weight. The constraint $T 1$ imposes a minimum SINR $\gamma_{\text {min }}$ needed per user. Constraint $T 2$ ensures that the sum-power consumption of all beams does not exceed the total power of the system $P_{\text {total }}$. Moreover, the constraint $T 3$ imposes a limitation for the power allocation per beam to be within the maximal allowed beam power, i.e. $P_{\max }$. In addition, $T 4$ and $T 5$ indicate that the respective carrier activation variables $x_{k}[i]$ and $y_{k}$ are binary. T6 indicates that the carrier assignment impacts the total number of active carriers. The constraint $T 7$ allow power to be allocated for $x_{k}[i]=1$. Finally, $T 8$ prohibits negative power allocation.

\section{DEMAND-AWARE POWER AND BANDWIDTH MINIMIZATION}

The unmet system capacity, the non-linearity of $\gamma_{i, k}\left(\mathbf{x}_{k}, \widetilde{\mathbf{p}}_{k}\right)$ as well as the integer nature of $x_{k}[i]$ and $y_{k}$ makes the problem (4) non-convex. Therefore, problem (4) is a mixed-integer non-convex optimization problem, for which it is difficult to find the optimal solution. Furthermore, to find the optimal values of $y_{k}$ and $\mathbf{x}_{k}$ an exhaustive search is required, which is computationally expensive even for problems with low dimension. Hence, to solve the problem, we first designed a suboptimal carrier allocation procedure where each carrier is allocated sequentially until the required capacity is provided to a user. Then, problem (4) with the above carrier allocation procedure reduces to a power allocation problem. We will show later how to address the power allocation problem. For $\mathbf{x}_{k}$ and $y_{k}$, we apply the following carrier allocation procedure:

$$
\begin{gathered}
y_{m}=\left\{\begin{array}{cc}
0, & \sum_{k=1}^{m-1} C_{i, k} \geq D_{i}, \forall_{i} \\
1, & \text { Otherwise }
\end{array}\right. \\
x_{m}[i]=\left\{\begin{array}{cc}
y_{m}, & \sum_{k=1}^{m-1} C_{i, k}<D_{i}, \\
0, & \text { Otherwise }
\end{array}\right. \\
C_{i, k}=B_{s c} \log _{2}\left(1+\gamma_{i, k}\left(\widetilde{\mathbf{p}}_{k}, \mathbf{x}_{k}\right)\right),
\end{gathered}
$$

where (5) describes the $m$ th carrier is available for transmission only if the previous $y_{m-1}$ carriers are not enough to satisfy the demand. This happens when the offered capacity to any beam is below the required demand. Furthermore, (6) states that the $m$ th carrier only available for beam $i$ whenever the offered capacity is less than the requested demand. Then, using (5) and (6) as well as introducing an upper bound slack variable $t_{i}$ to the unmet system capacity i.e. $1-C_{i} / D_{i} \leq t_{i}$, then the problem in (4) reduces to 


$$
\begin{array}{ll}
\underset{t_{i}, \forall_{i}, \widetilde{\mathbf{p}}_{k}, \forall_{k}}{\operatorname{minimize}} & \sum_{i=1}^{N} t_{i}+\sum_{i=1}^{N} \sum_{k=1}^{K} \widetilde{p}_{k}[i] \\
\text { s.t. } & T 1, T 2, T 3, T 7, T 8 \\
& T 9: 1-C_{i} / D_{i} \leq t_{i}, \forall_{i}, \\
& T 10: t_{i} \geq 0, \forall_{i},
\end{array}
$$

The problem in (8) is a power allocation problem that can be solved iteratively using quadratic transform, where the non-convex fractional part of the capacity is linearized [18]. Accordingly, the quadratic transform of $C_{i}$ is given by

$$
\begin{array}{r}
\widetilde{C}_{i}\left(\gamma_{i, k}\left(\mathbf{x}_{k}, \widetilde{\mathbf{p}}_{k}\right)\right)=\sum_{k=1}^{K} \log _{2}\left(1+\widetilde{\gamma}_{i, k}\left(\mathbf{x}_{k}, \widetilde{\mathbf{p}}_{k}\right)\right) \\
\left.\widetilde{\gamma}_{i, k}\left(\mathbf{x}_{k}, \widetilde{\mathbf{p}}_{k}\right)\right)=2 z_{i, k} \sqrt{g_{i, k}[i] x_{k}[i] \widetilde{p}_{k}[i] P_{\text {total }}} \\
-z_{i, k}^{2}\left(\sum_{j=1, j \neq i}^{N} g_{i, k}[j] x_{k}[i] p_{k}[j] P_{\text {total }}+\sigma^{2}\right) \\
z_{k}[i]=\frac{\sqrt{g_{i, k}[i] x_{k}[i] \widetilde{p}_{k}^{l}[i] P_{\text {total }}}}{\sum_{j=1, j \neq i}^{N} g_{i, k}[j] x_{k}[i] \widetilde{p}_{k}[j] P_{\text {total }}+\sigma^{2}}
\end{array}
$$

where $z_{k}[i]$ is the quadratic transform variable for each carrier and $\widetilde{p}_{k}^{l}[i]$ is the previous value of $\widetilde{p}_{k}[i]$. Finally, we re-write (8) as

$$
\begin{array}{ll}
\underset{\substack{\mathbf{p}_{k}, \forall_{k} \\
\text { s.t. }}}{\operatorname{minimize}} & \sum_{i=1}^{N} t_{i}+\sum_{i=1}^{N} \sum_{k=1}^{K} \widetilde{p}_{k}[i] \\
& T 1, T 2, T 3, T 7, T 8, T 10 \\
& T 9: D_{i}-\widetilde{C}_{i}\left(\widetilde{\gamma}_{i, k}\left(\mathbf{x}_{k}, \widetilde{\mathbf{p}}_{k}\right)\right) \leq t_{i}, \forall_{i}
\end{array}
$$

The (12) is now convex and can be solved using the available tools of convex optimization, such as CVX [19]. The proposed solution is described using the algorithmic notation in Algorithm 1. First, we initially assign equal power to $\tilde{p}_{k}^{l}[i]$. Furthermore, we initialize $C_{i}=0, y_{k}$ and $\mathbf{x}_{k}$ to zero. Then, the algorithm starts to update $y_{m}$ and $x_{m}[i]$ according to (5) and then (6), respectively. Hence, for $m=1$, the new updated value are $y_{1}=1$ and $x_{1}[i]=1$. Then, the algorithm solves (12) and updates the value of $\widetilde{p}_{k}^{l}[i]$ by the new values of $\widetilde{p}_{k}[i]$. Subsequently, the algorithm updates the values of $z_{k}[i], y_{m}$ and $x_{m}[i]$ within the loop ${ }^{1}$. The algorithm stops when the approximation of the quadratic form becomes accurate, i.e. the respective variable $z_{k}[i]$ does not change much within one iteration. Hence, the stopping condition is set as $\sum_{i=1}^{N} \sum_{k=1}^{K}\left|z_{k}[i]-z_{k}^{l}[i]\right| \leq 10^{-4}$, where $z_{k}^{l}[i]$ is the previous value of $z_{k}[i]$.

\footnotetext{
${ }^{1}$ Note that we are updating the values $y_{m}$ and $x_{m}[i]$ according to (5) and then (6) within the power allocation loop of algorithm 1. Hence, we avoid re-running the algorithm for new updates of carriers. Consequently, we reduce the computational time.
}

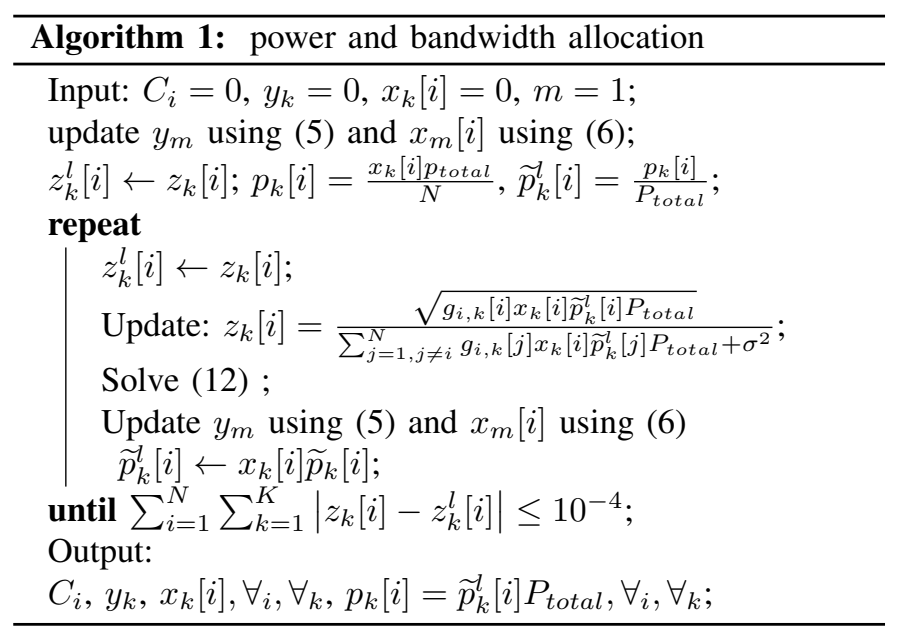

\section{Simulation Results}

This section evaluates the performance of the proposed Demand-Aware Power and Bandwidth Minimization (DAPBM) scheme. The main simulation parameters are summarized in Table I. We consider $N=100$ beams to cover a geographic area of Europe as shown in Fig. 1. The beam pattern is provided by the European Space Agency (ESA) and generated assuming a Direct Radiating Antenna (DRA), with 750 elements spaced by $5 \lambda$. Furthermore, the channel power gain for the $i$ th user from the $i$ th beam over the $k$ th carrier is given by $g_{i, k}[i]=\left|\frac{\sqrt{G_{R} G_{i}[i]}}{4 \pi \frac{d_{i}}{\lambda}}\right|^{2}$, where $G_{R}$ is the user terminal antenna gain, $G_{i}[i]$ denotes the gain from the $i$ th beam towards the $i$ th user and $d_{i}$ is the slant range between the satellite and the $i$ th user.

The results have been obtained with $M=100$ Monte Carlo runs. In each run, a user location is selected randomly from the considered beam coverage area. An example of user locations for a single realization is shown in Fig. 1. For comparison, we considered reference [10] and [16] as benchmark schemes. In addition, we considered that all users have the same demand $D_{i}=D, \forall i$, and we vary the value of $D$ between $100 \mathrm{Mbps}$ to $900 \mathrm{Mbps}$.

TABLE I

SYSTEM PARAMETERS

\begin{tabular}{|c|c|}
\hline Parameter & Value \\
\hline Satellite Orbit & $13^{\circ} \mathrm{E}$ \\
\hline Number of beams $(N)$ & 100 \\
\hline Number of carriers $(K)$ & 20 \\
\hline System Bandwidth $\left(B_{\text {tot }}\right)$ & $500 \mathrm{MHZ}$ \\
\hline Sub-carrier bandwidth $\left(B_{S C}\right)$ & $25 \mathrm{MHz}$ \\
\hline Noise power $\left(\sigma_{k}^{2}[i]\right)$ & $-130 \mathrm{dBW}$ \\
\hline Minimum SINR $\left(\gamma_{\min }\right)$ & $-2.2 \mathrm{~dB}$ \\
\hline Max. beam gain $\left(G_{i}[j]\right)$ & $51.8 \mathrm{dBi}$ \\
\hline User antenna gain $\left(G_{R}\right)$ & $39.8 \mathrm{dBi}$ \\
\hline Total Power of the system $\left(P_{\text {total }}\right)$ & $1000 \mathrm{~W}$ \\
\hline Maximum power for each beam $\left(P_{\max }\right)$ & $100 \mathrm{~W}$ \\
\hline
\end{tabular}




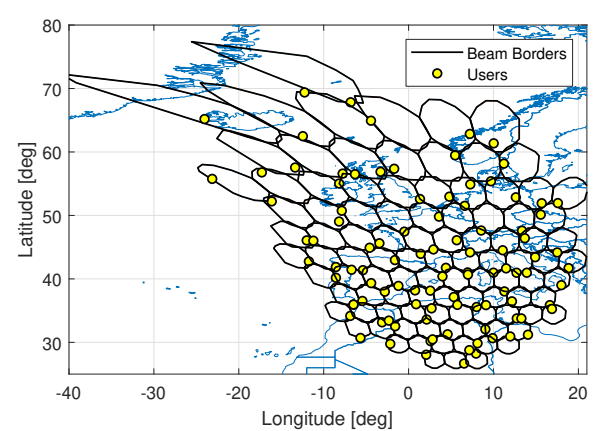

Fig. 1. User distribution for $N=100$ beam

\section{A. Convergence analysis}

The convergence of Algorithm 1 for different value of $D$ is shown in Fig. 2. For the convergence analysis we observe the objective function of (12) i.e. $F(t, \widetilde{p})=\sum_{i=1}^{N} t_{i}+$ $\sum_{i=1}^{N} \sum_{k=1}^{K} \widetilde{p}_{k}[i]$ at different iterations. We observed that the proposed Algorithm needs 6 iterations and 20 iterations to converge to the minimum value required to satisfy $100 \mathrm{Mbps}$ and $400 \mathrm{Mbps}$ demand per beam, respectively. Moreover, faster convergence is observed for lower demands in general, since fewer carriers are required.

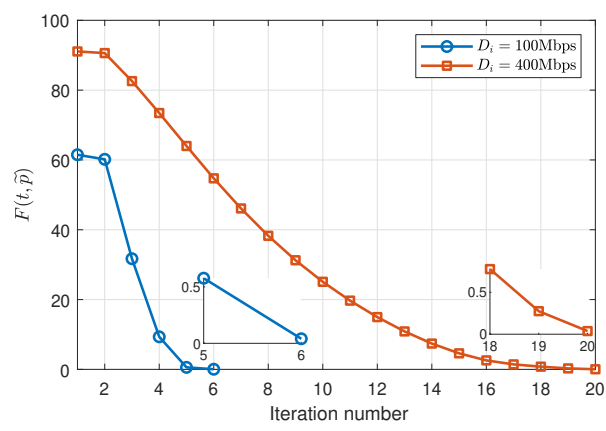

Fig. 2. Convergence analysis of Algorithm 1

\section{B. Computational Complexity Analysis}

The optimization (12) has $K(N+1)$ decision variables and $2 N K+3 N+1$ convex constraints. Assuming that the Algorithm 1 needs $M$ iterations to converge. Hence, the approximate Big- $\mathcal{O}$ complexity is given by $\mathcal{O}\left(M K^{3}(N+\right.$ $\left.1)^{3}(2 N K+3 N+1)\right) \approx \mathcal{O}\left(M(K N)^{4}\right)$ [20]. Therefore, the $\mathrm{Big}-\mathcal{O}$ of the proposed algorithm is a polynomial function that can efficiently run on computers in polynomial time. The actual computational time depends on the computer processors. For instance, using four $2.11 \mathrm{GHz}$ cores and $16 \mathrm{~GB}$ of $\mathrm{RAM}$, for a one-time resource allocation, the computational time for DAPBM, [16], and [10] is 5 minutes, 4 minutes, and 90 minutes, respectively. Hence, the computational time of the proposed (12) is less than the exponential cone optimization used in [10]. In contrast, [16] has less computational time than DAPBM. However, the performance of [16] is weaker than DAPBM as we will demonstrate in the next section.

\section{Demand matching}

In this section, we studied the demand satisfaction of the DAPBM and benchmark schemes. We compare the demand satisfaction using the following performance indicators

1) Average satisfaction index (ASI)

$$
\mathrm{ASI}=\frac{1}{N M} \sum_{m=1}^{M} \sum_{n=1}^{N} \min \left(\frac{C_{i}}{D_{i}}, 1\right)
$$

2) Average Unmet System Capacity (AUSC)

$$
\text { AUSC }=\frac{1}{N M} \sum_{m=1}^{M} \sum_{n=1}^{N} \max \left(D_{i}-C_{i}, 0\right)
$$

Fig. 3a provides the ASI for the DAPBM and the benchmark schemes. We observed decreased ASI from $450 \mathrm{Mbps}$ for the proposed scheme and [10], whereas the starting decrease of ASI for [16] is from $100 \mathrm{Mbps}$. Moreover, at $500 \mathrm{Mbps}$, the DAPBM provides $99 \%$ demand satisfaction but [10] and [16] have $97.5 \%$ and $89 \%$ demand satisfaction, respectively. Furthermore, on average, the DAPBM can satisfy the demand of $450 \mathrm{Mbps}$ and $900 \mathrm{Mbps}$ per beam at $100 \%$ and $69 \%$ cases, respectively but not in the benchmark schemes. Therefore, the proposed scheme outperforms the benchmark schemes. Moreover, in [10], the radiation power used in some carrier frequencies is small. Hence, poor SINR is observed below the system requirement. Consequently, lower demand satisfaction is observed. However, in DAPBM, a minimum SINR constraint is included to avoid the result of poor SINR. Hence, we gain more demand satisfaction compared to [10]. For [16], an evolutionary algorithm is used to allocate the power and bandwidth, which can not guarantee the optimal solution. As a result, poor demand satisfaction is observed. In contrast, in DAPBM, higher demand satisfaction is obtained since it closely converges to the optimal solution.

Fig.3b depicts the AUSC performance of the DAPBM and the benchmark schemes. The AUSC is zero below 450Mbps, for DAPBM and [10]. In contrast, the AUSC is non zero for [16]. Moreover, since the proposed algorithm minimizes the power and the carrier while closely matching the demand, it provides no excess offered capacity. Consequently, the mismatch error is zero up to $450 \mathrm{Mbps}$. However, we observed above $450 \mathrm{Mbps}$ increase of the AUSC for all schemes. For instance, at $600 \mathrm{Mbps}$, the AUSC of DAPBM is $51 \mathrm{Mbps}$, whereas the AUSC of [10] and [16] is $61 \mathrm{Mbps}$ and $132 \mathrm{Mbps}$, respectively. Hence, the system resource is not enough to accommodate higher demands. Nevertheless, precoding can be added on the top of the proposed scheme to improve demand satisfaction. Generally, the proposed scheme performed well for lower and medium demands. 


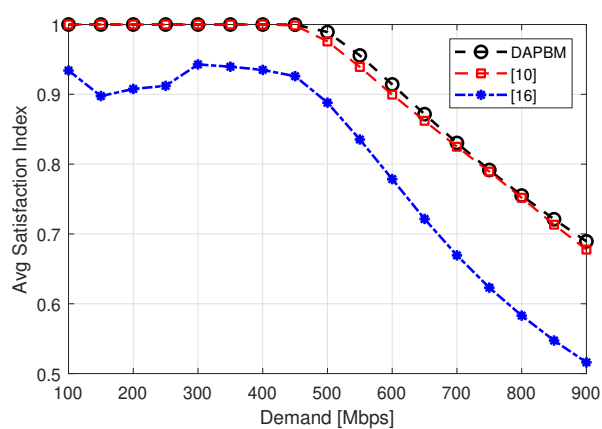

(a)

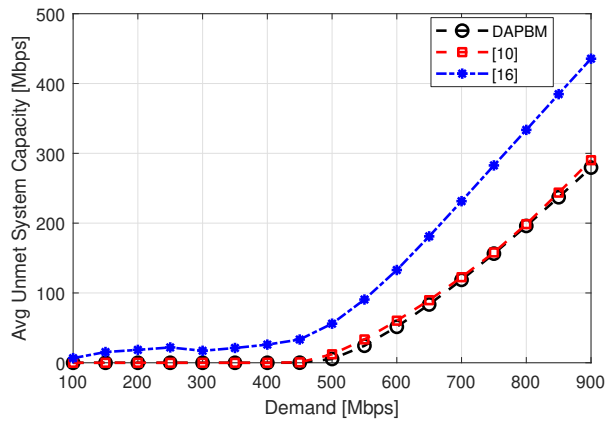

(b)

Fig. 3. Comparison of DAPBM and benchmark schemes.: (a) ASI; (b) AUSC

\section{Bandwidth and power consumption}

In this section, we compare the bandwidth and the power consumption of all schemes. We use the following key performance indicators:

\section{1) Average used power (AUP)}

$$
\mathrm{AUP}=\frac{1}{M} \sum_{m=1}^{M} \sum_{i=1}^{N} \sum_{k=1}^{K} p_{k}[i] .
$$

2) Average used bandwidth (AUB)

$$
\mathrm{AUB}=\frac{B s c}{M} \sum_{m=1}^{M} \sum_{k=1}^{K} y_{k} .
$$

Fig. 4a describes the AUB of all schemes. We have observed that the bandwidth utilization of the DAPBM is more efficient than the benchmark schemes. For instance, at $100 \mathrm{Mbps}$ and $400 \mathrm{Mbps}$, respectively, the proposed scheme allocates $26 \%$ and $90 \%$ of the system bandwidth while [10] and [16] allocates $100 \%$ of the system bandwidth. Interestingly, the proposed algorithm allocates the bandwidth properly with respect to demand. For lower demands, a small portion of the bandwidth is required. As the demand increases, the bandwidth allocation increases. Therefore, the DAPBM allocates the system bandwidth dynamically with a change of per beam demand. This is not possible in the case of the benchmark schemes. However, for higher demand, the DAPBM usage of bandwidth converges to the system bandwidth.

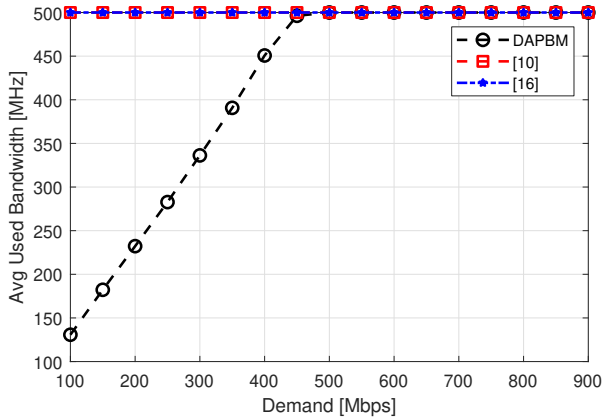

(a)

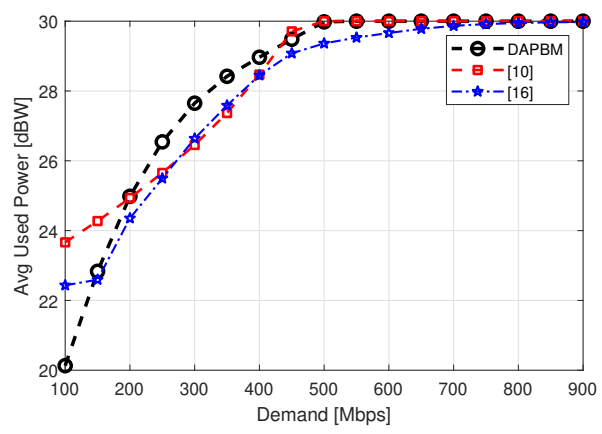

(b)

Fig. 4. Comparison of DAPBM and benchmark schemes: (a) AUB; (b) AUP.

The AUP in dBW of DAPBM and the benchmark schemes are shown in Fig. $4 \mathrm{~b}$. We observed that the proposed scheme has less power consumption for low demand compared with the benchmark schemes. For example, at $100 \mathrm{Mbps}$ the DAPBM is lower than [10] and [16] by $3 \mathrm{dBW}$ and $2 \mathrm{dBW}$, respectively. For medium demands, the DAPBM used slightly more power compared to [10]. However, this more power consumption is compensated by saving more bandwidth, see Fig.4a. Though [16] have lower power consumption than the proposed scheme, the power allocation is not efficient. Consequently, poor demand satisfaction is observed. Generally, for higher demands, all scheme converges to the total power of the system.

\section{E. Carrier allocation}

In this section, we compare the carrier allocation of the DAPBM scheme at different demands. We use the following performance indicators :

\section{1) Average used carriers (AUC)}

$$
\mathrm{AUC}=\frac{1}{M} \sum_{m=1}^{M} \sum_{k=1}^{K} y_{k} .
$$

\section{2) Average unused carriers (AUNC)}

$$
\mathrm{AUNC}=\frac{1}{M} \sum_{m=1}^{M}\left(K-\sum_{k=1}^{K} y_{k}\right) .
$$

Fig. 5 shows the AUC and AUNC of the proposed scheme. At low demand, the DAPBM utilizes fewer carriers. For instance, 
at $100 \mathrm{Mbps}$, the AUNC is 15, and the AUC is 5. Furthermore, we observed that the AUC increases with respect to demand. However, the AUNC decreases when the demand increases. Generally, the proposed algorithm saves more unused carriers for demand below 400Mbps. Accordingly, those carriers can be applied to other services at the same time while satisfying the current system services. Finally, thanks to the proposed scheme, we can satisfy at most $400 \mathrm{Mbps}$ per beam demand while saving $10 \%$ of the carriers.

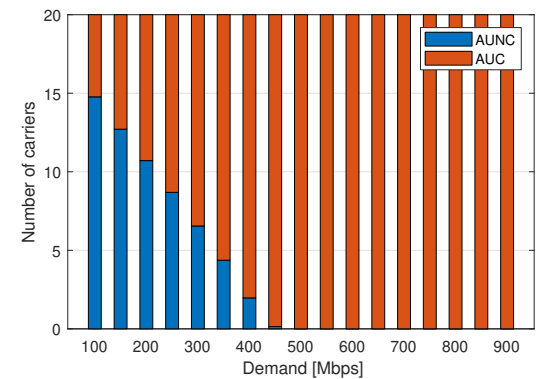

Fig. 5. Carrier allocation of DAPBM

\section{CONCLUSIONS}

In this paper, we proposed a novel method of Power and Bandwidth Minimization for Demand-Aware GEO Satellite Systems. The formulated optimization problem is non-convex in general. Hence, we use demand-aware carrier assignment with power allocation to solve the problem iteratively. The carrier assignment is done sequentially with the goal to not occupy additional carriers, if the demand is already satisfied. The power allocation remains a non-convex problem, which is solved using linearization and iterative update. The proposed scheme is compared with the benchmark schemes via extensive numerical simulations. we observed that the proposed scheme provides better performance in terms of demand satisfaction and bandwidth efficiency.

\section{REFERENCES}

[1] O. Kodheli, E. Lagunas, N. Maturo, S. K. Sharma, B. Shankar, J. F. M. Montoya, J. C. M. Duncan, D. Spano, S. Chatzinotas, S. Kisseleff, J. Querol, L. Lei, T. X. Vu, and G. Goussetis, "Satellite communications in the new space era: A survey and future challenges," IEEE Communications Surveys Tutorials, vol. 23, no. 1, pp. 70-109, 2021.

[2] "SES-17 satellite, more information available at:" https://www.ses.com/press-release/ses-and-thales-unveil-nextgeneration-capabilities-onboard-ses-17, accessed: 2021-3.
[3] "Eutelsat Quantum satellite, more information available at:", https://spacenews.com/intelsat-to-market-half-of-capacity-on-eutelsatsquantum-satellite/, accessed: 2021-3.

[4] S. Kisseleff, E. Lagunas, T. S. Abdu, S. Chatzinotas, and B. Ottersten "Radio resource management techniques for multibeam satellite systems," IEEE Communications Letters, pp. 1-1, 2020.

[5] N. K. Srivastava and A. K. Chaturvedi, "Flexible and Dynamic Power Allocation in Broadband Multi-Beam Satellites," IEEE Communications Letters, vol. 17, no. 9, pp. 1722-1725, Sep. 2013.

[6] A. Destounis and A. D. Panagopoulos, "Dynamic Power Allocation for Broadband Multi-Beam Satellite Communication Networks," IEEE Communications Letters, vol. 15, no. 4, pp. 380-382, April 2011.

[7] A. I. Aravanis, B. Shankar M. R., P. Arapoglou, G. Danoy, P. G. Cottis, and B. Ottersten, "Power Allocation in Multibeam Satellite Systems: A Two-Stage Multi-Objective Optimization," IEEE Transactions on Wireless Communications, vol. 14, no. 6, pp. 3171-3182, June 2015.

[8] J. P. Choi and V. W. S. Chan, "Optimum power and beam allocation based on traffic demands and channel conditions over satellite downlinks," IEEE Transactions on Wireless Communications, vol. 4, no. 6, pp. 2983-2993, Nov 2005.

[9] T. Qi and Y. Wang, "Energy-efficient power allocation over multibeam satellite downlinks with imperfect CSI," in International Conference on Wireless Communications Signal Processing, Oct 2015, pp. 1-5.

[10] C. N. Efrem and A. D. Panagopoulos, "Dynamic Energy-Efficient Power Allocation in Multibeam Satellite Systems," IEEE Wireless Communications Letters, vol. 9, no. 2, pp. 228-231, 2020.

[11] U. Park, H. W. Kim, D. S. Oh, and B. J. Ku, "Flexible Bandwidth Allocation Scheme Based on Traffic Demands and Channel Conditions for Multi-Beam Satellite Systems," in IEEE Vehicular Technology Conference, Sep. 2012, pp. 1-5.

[12] Heng Wang, Aijun Liu, Xiaofei Pan, and Luliang Jia, "Optimal bandwidth allocation for multi-spot-beam satellite communication systems," in Proceedings 2013 International Conference on Mechatronic Sciences, Electric Engineering and Computer (MEC), 2013, pp. 2794-2798.

[13] J. Lei and M. A. Vázquez-Castro, "Joint Power and Carrier Allocation for the Multibeam Satellite Downlink with Individual SINR Constraints," in IEEE International Conference on Communications, May 2010, pp. $1-5$

[14] $\_$, "Multibeam satellite frequency/time duality study and capacity optimization," Journal of Communications and Networks, vol. 13, no. 5 , pp. 472-480, 2011.

[15] A. Paris, I. Del Portillo, B. Cameron, and E. Crawley, "A Genetic Algorithm for Joint Power and Bandwidth Allocation in Multibeam Satellite Systems," in IEEE Aerospace Conference, March 2019, pp. $1-15$.

[16] G. Cocco, T. de Cola, M. Angelone, Z. Katona, and S. Erl, "Radio Resource Management Optimization of Flexible Satellite Payloads for DVB-S2 Systems," IEEE Transactions on Broadcasting, vol. 64, no. 2, pp. 266-280, Jun. 2018

[17] T. S. Abdu, E. Lagunas, S. Kisseleff, and S. Chatzinotas, "Carrier and power assignment for flexible broadband geo satellite communications system," in 2020 IEEE 31st Annual International Symposium on Personal, Indoor and Mobile Radio Communications, 2020, pp. 1-7.

[18] K. Shen and W. Yu, "Fractional programming for communication systems-part i: Power control and beamforming," IEEE Transactions on Signal Processing, vol. 66, no. 10, pp. 2616-2630, 2018.

[19] M. Grant and S. Boyd, "CVX: Matlab software for disciplined convex programming, version 2.1," http://cvxr.com/cvx, Mar. 2014.

[20] P. Gahinet, A. Nemirovski, A. J. Laub, and M. Chilali, LMI Control Toolbox Users Guide. USA: MathWorks, 1995. 